\title{
EL DERECHO A SUFRAGIO DE LOS MENORES DE EDAD: CAPACIDAD Y EDAD ELECTORAL ${ }^{* *}$
}

\section{The right to vote for minors: electoral capacity and electoral age}

\author{
PABLO MARSHALL
}

Universidad Austral de Chile

\begin{abstract}
RESUMEN
Este artículo ofrece un breve marco conceptual para entender cómo se debe atribuir el derecho a sufragio en una comunidad democrática, para luego explorar una de las principales razones para fijar la edad electoral en los 18 años. El texto se ocupa detenidamente del popular argumento de que los menores de edad carecen de capacidad electoral, para lo que analiza críticamente la consistencia de las reglas sobre esta materia y explora las alternativas a la fijación de una edad mínima para votar. El artículo concluye que hay buenas razones para pensar que podría rebajarse a los 16 años, principalmente porque la responsabilidad legal es consistentemente reconocida por el sistema jurídico a esa edad. Ello sería un poderoso argumento en el contexto de una democracia que atribuye derechos de participación política como consecuencia del reconocimiento de la personalidad deliberativa, en la que también se funda la atribución de la capacidad legal asociada al concepto de sujeto de derecho.
\end{abstract}

Palabras clave: derecho a sufragio, menores, capacidad electoral

\begin{abstract}
This article offers a brief conceptual framework for understanding the allocation of the right to vote in a democratic community. It explores one of the main reasons for setting the electoral age at 18 years. The article focuses on the popular argument that minors lack electoral capacity, critically analyzing the consistency with which rules on electoral capacity are applied, and exploring alternatives to the use of a minimum age restriction. It concludes that there are good reasons to believe that this age could be lowered 16 years, as the capacity of individuals is already clearly recognized by the legal system at that age. This may be a powerful argument in support of the hypothesis that a democracy attributes democratic rights as a consequence of the recognition of deliberative personality, on which the attribution of legal capacity associated with the legal subject is also based.
\end{abstract}

Key words: right to vote, minors, electoral capacity

\footnotetext{
El autor agradece los comentarios de los profesores Juan Pablo Mañalich (Universidad de Chile), Rocío Lorca (Universidad de Chile) y Álvaro Núñez (Universidad Austral de Chile) a alguno de los borradores de este artículo. Especiales agradecimientos para uno de los árbitros anónimos, quien formuló incisivos y generosos comentarios que ayudaron a mejorar considerablemente el artículo.

* $\quad$ Este artículo se inscribe en el proyecto Fondecyt 11150299 (Una teoría sobre la función del derecho a sufragio: participación y reconocimiento) del que el autor es investigador principal.
} 


\section{INTRODUCCIÓN}

Para autorizar o prohibir diferentes comportamientos y actividades a los menores de edad, se tiende a evaluar tanto las circunstancias sociales de las personas involucradas, como sus capacidades cognitivas. En relación al primer elemento, por ejemplo, las razones más importantes para prohibir el trabajo infantil no se relacionan con la capacidad de los niños para trabajar, sino con la inconveniencia de participar en tales labores teniendo en cuenta sus vulnerabilidades y sus necesidades específicas de protección, desarrollo y educación. Tomar en consideración la capacidad cognitiva de los menores, por otro lado, es tremendamente problemático, dado que se traslapan factores biológicos con elementos socialmente construidos y, por tanto, dependientes de la experiencia individual. No obstante, resulta difícil ignorar la significativa diferencia cognitiva que existe entre un niño de tres años y un adolescente de 16.

Es reconocido por los psicólogos del desarrollo, por ejemplo, que los seres humanos no nacen con la capacidad de hacer elecciones racionales, sino que la incrementan con la edad. Sin embargo, es también advertido que no todos lo hacen de una manera similar o al mismo tiempo. En este sentido, incluso cuando la regulación legal trate de ser lo más acuciosa y específica al determinar la edad que habilita a las personas a desarrollar ciertas actividades, siempre existirá un factor de arbitrariedad que implicará falsos negativos y positivos (Cowden 2012: 363-5).

El problema de la compleja relación entre edad y capacidades es particularmente interesante en la discusión sobre la fijación de la edad electoral. Este es un debate vigente que informa la discusión legislativa y, en ciertos casos, el establecimiento de normas electorales que permiten la participación de menores de 18 años en votaciones. Así, por ejemplo, en 2007, Austria se constituyó en el primer miembro de la Unión Europea en rebajar la edad electoral a los 16 años, en tanto que España, Italia y el Reino Unido han comenzado a debatir medidas similares en sus respectivos parlamentos. En Latinoamérica, Nicaragua (1984) y Brasil (1988) fueron no solo los precursores regionales, sino que mundiales de esta medida. Les siguieron Ecuador (2008) y Argentina (2012). Al mismo tiempo, su discusión ya se ha iniciado en Bolivia, Chile, Venezuela y Uruguay.

Este artículo analiza la exclusión de la participación electoral de los menores de edad en razón de su supuesta falta de capacidad electoral. Esta debe entenderse como aquellas capacidades individuales necesarias para participar en elecciones y plebiscitos de manera competente y autónoma. La noción de capacidad electoral expresa una demanda en la configuración de la figura del elector como un sujeto racional e informado y a ella subyace la idea de que las elecciones requieren, para su correcto funcionamiento, tal clase de participación.

Para este análisis, se tomará como punto de referencia la idea actualmente en boga de rebajar la edad electoral desde los 18 a los 16 años, lo que se concluye afirmativamente al confirmase que existen buenas razones para aceptar 
dicha modificación. El artículo se estructura en tres secciones. En la primera se contextualiza la discusión, evaluando la noción de sufragio universal y presentando algunas de las razones que se suelen esgrimir para excluir el voto de los menores de edad. La siguiente se centra en la idea de la falta de capacidad electoral en los menores de 18 años, analizando los problemas que la afectan y revisando varias soluciones insatisfactorias a dichos problemas. Finalmente, se presenta una solución que parte de cierta homologación entre capacidad electoral y jurídica o legal. Así, se sostiene que, en una democracia, la capacidad legal y la electoral y política están íntimamente ligadas y, por tanto, deben reconocerse derechos de participación a los sujetos de quienes se espera una intervención competente en la vida jurídica.

\section{EL SUFRAGIO DE LOS MENORES DE EDAD}

\section{Sufragio universal como una carga de argumentación}

La tarea de determinar quiénes deben ejercer el derecho a votar en una democracia está directamente ligada a la elaboración de la idea de sufragio universal. Pese a que es invariablemente considerado entre los criterios para catalogar como democrático a un régimen político, cuando se observa quienes están legalmente habilitados para sufragar no existe país alguno donde todos sus habitantes se cuenten entre los electores. Pero, si no es efectivamente universal ¿qué es lo que implica la universalidad en este caso?

La lectura que Beckman (2007) hace de la literatura sobre el punto sostiene que la idea de sufragio universal dependerá de la noción de inclusión democrática que se considere parte del concepto de democracia. Las formulaciones minimalista (que solamente se requiere elecciones competitivas, sin considerar como necesaria la inclusión de ningún grupo de personas), convencionalista (que exige la participación de todos los no usualmente excluidos por la práctica electoral comparada) o maximalista (que toma en cuenta el universo de los residentes del país sin distinción, para luego considerar a los que son excluidos del sufragio como un déficit democrático prima facie), ofrecen diferentes respuestas a la pregunta sobre quién debe votar en una democracia. También permiten aproximaciones más o menos críticas a los casos en que ciertas personas son marginadas de las elecciones (Beckman 2007: 34-40).

Si se asume el punto de vista normativo de las formulaciones maximalistas, el principio de sufragio universal no es concebido como una descripción del universo electoral, sino una regla de carga de la argumentación (o la determinación de quien tiene el deber de justificar) a la hora de determinar quiénes sirven como electores en una comunidad política dada (Beckman 2009). Dentro de esta hipótesis, es fundamental distinguir entre una democracia excluyente y una inclusiva. Desde la perspectiva excluyente, la inclusión en el círculo de los electores debe justificarse por medio de argumentos sobre la 
contribución que los potenciales miembros del electorado podrían realizar al proceso político. Así, por ejemplo, este enfoque podría evaluar la incorporación de los menores de edad en términos de su potencial impacto en la participación política general. Si la inclusión de los menores puede proveer un input de legitimación basado en mayor participación electoral, su inclusión podría estar justificada. Si, por el contrario, se descubre que la participación general decrecerá como consecuencia de su inclusión y que, por tanto, también lo hará eventualmente la legitimidad política del gobierno, no debería incluírselos entre los electores (López-Guerra 2010: 123-4; Wagner et al. 2012: 372-383). En contraste, para una democracia inclusiva, el sufragio debe ser atribuido prima facie a todos los habitantes, y es la exclusión de un sujeto o grupo de sujetos lo que debe ser justificado. Así, la exclusión de ciertos grupos del electorado podría ser compatible con el principio de sufragio universal, siempre y cuando esté debidamente justificada. Para ello, es imprescindible que la excepción y su justificación no cuestionen el principio de sufragio universal mismo como regla general. No puede, por ejemplo, pretender sustituir la regla general de que, en principio, todos deben poder votar, por una norma de meritocracia según la cual, por principio, solo algunos deberían poder sufragar en virtud de sus particulares aptitudes (Olsson 2008: 58).

Los argumentos que se presentan en este artículo transcurren bajo el marco conceptual y argumentativo de esta comprensión del principio de sufragio universal, esto es, bajo una concepción maximalista e inclusiva. En este contexto, la exclusión electoral de todos los grupos que se encuentran comúnmente excluidos de la participación debe ser justificada (Beckman 2009; Lopez-Guerra 2014). Entre ellos están los extranjeros, cuya exclusión se basaría en la ausencia de un vínculo de pertenencia a la comunidad política y eventuales conflictos de interés (Lopez-Guerra, 2014: cap. 4); los criminales y los presos, cuya exclusión no ha sido satisfactoriamente ofrecida (The Disenfranchisement of Ex-Felons 1989); las personas con alguna deficiencia mental (Beckman 2014); $y$, finalmente, los menores de edad.

\section{¿Por qué los menores de 18 años no pueden votar?}

Históricamente se han ofrecido numerosas razones para negar el voto a los menores de edad. En general, dado que los defensores de la mantención de la actual edad electoral se encuentran bajo el amparo del status quo, no es fácil encontrar articulaciones razonadas para dicha exclusión. Excepcionalmente, durante la reforma electoral austríaca, se desarrolló una revisión de esta clase de razones, la que llevó a rebajar la edad electoral hasta los 16 años (Wintersberger 2007). Se afirmó que los jóvenes:

1. votan en forma distinta a los adultos,

2. su voto iría a partidos radicales,

3. no están interesados en votar, 
4. tampoco se les permite votar en otros países,

5. tienen sus propias instituciones juveniles en las que pueden participar,

6. son inmaduros y

7. son manipulables.

Un breve análisis de estas razones dejará expuesta la necesidad de centrar la discusión en la cuestión de la capacidad electoral. Debe llamarse la atención sobre lo inaceptable de sostener (1) y (2) en un régimen democrático, pues el presumible contenido del voto, cualquiera sea el contenido de esa preferencia, no puede ser considerado una razón válida para excluir a alguien de la participación electoral (Beckman 2009: 141). Teóricamente, este punto está ligado a los principios de neutralidad estatal y pluralismo político esenciales en una democracia (Winkler 1993: 353-358). Desde una perspectiva práctica, el descarte de electores solo se ha prestado para abusos de la mayoría sobre las minorías políticas (Ewald 2004: 119-24). La tercera razón no merece tomarse seriamente. Por un lado, los derechos sirven justamente al propósito de hacer inviolable la esfera de acción que garantizan frente a argumentos como este (Dworkin 1978: 430-3; Habermas 1996: 258). Por otro lado, la apatía política de los jóvenes puede deberse, al menos parcialmente, a la falta de respeto que se tiene hacia sus opiniones, algo que el derecho a sufragio podría corregir (Munn 2012: 155-6). En contra de la quinta razón, el que los jóvenes tengan espacios de participación propios, no debería excluirlos de los mecanismos de participación general. Por el contrario, su capacidad de asociarse y contribuir en dichas instancias debería verse como una razón para considerar que pueden hacerlo en procesos reservados hasta ahora a los mayores. Finalmente, en relación al cuarto factor, ya se ha mencionado que existe una tendencia global a discutir y legislar en favor de la inclusión de los mayores de 16 años.

Las razones seis y siete, por el contrario, parecen coincidir con las ideas que la literatura especializada discute actualmente al tratar el sufragio de los menores de edad. Por un lado, este grupo sería excluido dado que sus individuos carecen de las capacidades adecuadas para asumir la responsabilidad que el voto involucra, cualquiera sea el contenido de estas capacidades (por ejemplo, no ser inmaduros y manipulables) (Blais et al. 2001: 43). Por otro lado, su participación podría arruinar el contenido de la elección dada su falta de capacidades (Olsson 2008: 62). El primer argumento, llamado de la capacidad electoral, apunta a identificar cierta falta de aptitud para ejercer el derecho a sufragio en ciertos sujetos, en este caso los menores de edad. Por el contrario, el segundo argumento, que puede ser llamado del impacto electoral, mira a las consecuencias de la participación de dichos sujetos en el proceso político y, en particular, en las elecciones. No obstante ser distintos, ambos argumentos son simbióticos. Si se demuestra que la incompetencia de los adolescentes no tiene una repercusión negativa en los resultados del proceso electoral, las razones para preocuparse por su capacidad se ven considerablemente limitadas, tienden a desaparecer o se vuelven irrelevantes. En contraste, si se comprueba que los mayores de 16 años cuentan con la capacidad para participar en las elecciones, el argumento de que 
su participación arruinaría el resultado electoral no puede sostenerse sin caer en los problemas identificados para (1) y (2). En lo que sigue este trabajo, se enfoca en la cuestión de la capacidad electoral y deja de lado cuestiones relacionadas con el impacto electoral del voto de los menores de edad.

Esto no sugiere que no haya otros posibles argumentos para justificar la fijación de la edad electoral que pueden superponerse o bien ser independientes del argumento de la capacidad política, sin embargo, este artículo se limita a examinar este supuesto y, por ello, deja de lado otras consideraciones que pueden parecer relevantes, por ejemplo, la posible influencia negativa que la participación electoral podría implicar en la vida de los niños (Beckman 2009: 114-9). Para ser claro, este texto solo examina la vinculación entre la idea de capacidad electoral y la fijación de una edad mínima electoral.

\section{LA CAPACIDAD ELECTORAL Y LA EXCLUSIÓN DE LOS MENORES DE EDAD}

Esta sección busca realizar un breve análisis de cómo, la implementación institucional de la noción de capacidad electoral, justifica excluir del derecho a sufragio a los menores de edad. Para ello, (1) se exponen algunas nociones de lo que podría entenderse como incluido en la capacidad electoral. Estas nociones (2) se contrastan con evidencia empírica disponible y con argumentos teóricos, para luego concluir que ellas presentan importantes debilidades a la hora de explicar la exclusión de los menores de edad. A continuación, (3) se evidencia la falta de coherencia de las reglas electorales que implementan la idea de capacidad electoral, respecto de otros casos que se encuentran en circunstancias similares a los menores. Finalmente, se revisan críticamente dos ideas alternativas a la edad electoral para abordar la cuestión de la capacidad: (4) utilizar un test para evaluarla en todos los ciudadanos y (5) rechazarla totalmente, otorgando derecho a voto a todos los menores de edad desde su nacimiento.

\section{La capacidad electoral como criterio para limitar el sufragio}

Como se ha afirmado, uno de los principales argumentos para justificar la exclusión de los menores de la participación electoral es que ellos carecen de ciertas capacidades. Ello, a su vez, implica una presunción de que quienes han cumplido los 18 años son capaces electoralmente hablando (Munn 2012: 141). No obstante lo extendido de este argumento en la literatura sobre el derecho a sufragio, poca atención se ha prestado a su justificación (López-Guerra 2010: 115). Es fundamental clarificar qué se quiere decir con capacidad electoral en este contexto. 
Para que la discusión tenga sentido, el concepto de 'capacidad' debe hacer referencia a alguna habilidad mental o conocimiento adquirido que posibilita participar en elecciones de manera competente. Esto es, una capacidad que los adultos habrían desarrollado durante su juventud y que estaría ausente, o en desarrollo, en los menores de edad.

Siguiendo a Lau (2012), pueden mencionarse tres diferentes criterios para determinar esta noción y que contribuirían a demostrar que los menores de 18 años deben ser excluidos del electorado.

El primero está ligado a la idea de racionalidad. El sufragio debe ser atribuido a aquellos que puedan ejercerlo haciendo uso de una capacidad general para tomar decisiones racionales. Esto no necesariamente implica que todos los adultos decidan racionalmente todo el tiempo, sino que ellos, en términos generales, tienden a hacerlo. Por ejemplo, tienden a ser exitosos en la selección de ciertos medios para alcanzar ciertos fines. Los menores, por otro lado, generalmente no son o no debieran estar involucrados en situaciones en que puedan demostrar que pueden actuar racionalmente. La capacidad de razonamiento, de acuerdo a esta propuesta, es la que estaría desarrollada totalmente cuando los jóvenes se vuelven adultos (Lau 2012: 862).

Una segunda concepción apunta al conocimiento político-teórico del sujeto en cuestión. Se trata del conocimiento y comprensión que un sujeto demuestra del proceso político en general y del sistema electoral en particular. En este sentido, el individuo debe ser consciente de la importancia de las decisiones que se están tomando, así como de la forma en que estas son tomadas. Por ejemplo, saber cuáles son los partidos políticos en competencia, cómo se sufraga o cuántos diputados componen el Parlamento, son indicadores que pueden ser usados para evaluar aquellos aspectos. En estos términos, los menores de edad muestran un menor conocimiento empírico de hechos relacionados con el funcionamiento del sistema político (Lau 2012: 862-3). Definido así, el conocimiento político es una noción considerablemente menos ambiciosa que la anterior y más fácil de medir. Sin embargo, es también más restrictiva.

Finalmente, la 'capacidad' puede ser entendida como la comprensión de asuntos políticos específicos y su relación con el sistema político y el proceso electoral. Este conocimiento político pragmático estaría dado por la posibilidad de favorecer una preferencia política mediante el acto del voto. Para ello, es necesaria una asociación de determinadas ideologías y propuestas políticas con ciertos partidos y candidatos. Por ejemplo, la comprensión de que si se vota por un candidato conservador se estará votando en contra de la despenalización del aborto, sería un buen indicador para medir este aspecto. Se sostiene que esta 'capacidad', tampoco estaría suficientemente desarrollada en los menores de 18 años (Lau 2012: 863-4). 


\section{Los problemas de la edad mínima para medir la capacidad electoral}

Estas tres concepciones de capacidad electoral presentan problemas a la hora de justificar la fijación de la edad electoral en los 18 años. En primer lugar, y en relación a la noción de racionalidad, no ha sido todavía demostrado quelos adultos, y en particular los adultos entre 18 y 30 años de edad, tengan una capacidad racional mayor que la de los adolescentes (Lau 2012: 862; Hart y Atkins 2011). Si se entiende por 'racional' el actuar siguiendo un plan de acción previamente establecido y que tiende a responder exitosamente a las expectativas esperadas, claramente es posible concluir que un niño de tres años lo es menos que un adulto. Sin embargo, esta conclusión no es posible cuando se comparan adultos con menores de 16 años. Hart y Atkins (2011) citan varios estudios empíricos recientes que demuestran justamente lo contrario. Por un lado, se ha concluido que la consolidación del desarrollo cognitivo de los seres humanos terminaría a los 16 años (Hart y Atkins 2011: 218). Por otro, si bien existen diferencias entre las estructuras neurológicas de los adolescentes y de los jóvenes-adultos, estas diferencias no resultan relevantes a la hora de tomar decisiones con efectos a largo plazo. Las desigualdades entre menores-adolescentes y jóvenes-adultos se observan en ámbitos discretos, como las relaciones entre pares y las actividades riesgosas, lo que no posibilitaría justificar una diferenciación a la hora de atribuir capacidad electoral (Hart y Atkins 2011: 219).

En segundo lugar, con respecto al conocimiento político —-teórico y pragmático-, no es sorpresa alguna que los menores de edad, excluidos de la participación electoral, no tengan un manejo equivalente al que los adultos han adquirido con el tiempo, principalmente a través de la misma práctica del sufragio. En este sentido, se puede predecir con relativa seguridad que, si se extiende el voto, por ejemplo, a los mayores de 16 años, su conocimiento sobre el sistema político y electoral mejorará sustancialmente (Lau 2012: 863). La noción de capacidad electoral como 'conocimiento' es, en cierta medida, manufacturada por la inclusión en el derecho a ejercer el voto. Por otro lado, investigaciones empíricas hablan de una falta de diferencias estadísticas relevantes en los niveles de conocimiento político (Wagner et al. 2012: 375-80) y que los mayores de 16 años están equipados con las mismas habilidades y conocimientos que los adultos (Hart y Atkins 2011: 212-3).

En último término, algunas investigaciones han mostrado diferencias efectivas en términos de conocimiento de jóvenes entre 16 y 18 años con el universo de los electores adultos. Es decir, los menores entre 16 y 18 años tendrían conocimientos (políticos generales y pragmáticos) más reducidos que los de adultos. Sin embargo, cuando se los compara solo con el grupo de los electores entre 19 y 20 años, tal tendencia desaparece e incluso la relación se invierte (Hart y Atkins 2011: 214). Así, si bien excluir a los menores de 18 contribuye a consolidar un electorado más informado respecto del que estadísticamente resultaría al incluirlos, el umbral de exclusión — de acuerdo a los indicadores de conocimiento- debería ser fijado en los 30 años, cuando los electores muestran 
un conocimiento político consolidado. Respecto a ese universo electoral, incluir a los jóvenes de entre 18 y 30 años solo contribuiría a la devaluación del conocimiento político promedio (Olsson 2008: 68).

En suma, la fijación de la edad electoral a los 18 años sería arbitraria en la medida que excluye, al menos respecto a los mayores de 16 años, un grupo de individuos que sí tienen capacidad electoral según los criterios expuestos. La concepción de la capacidad electoral según los criterios de racionalidad y de conocimiento político (teórico y pragmático), aunque necesarios, no serían suficientes para justificar la fijación de la edad electoral en los 18 años.

\section{Universalizando el argumento de la capacidad electoral}

La defensa de la capacidad electoral, como justificación para la exclusión de los menores, debe sortear una segunda dificultad: someterse a un test de universalidad. Dicha dificultad consiste en que, si se excluye a los menores de 18 años por una carencia de capacidad electoral, se debe estar dispuesto a excluir por las mismas razones a aquellos que superan esa edad y que carezcan de dicha capacidad, cualquiera sea el estándar que se use para evaluarla. Lau (2012) desarrolla esta idea y formula un argumento sobre la simetría de la capacidad electoral.

Lau sostiene que la edad electoral mínima es una presunción basada en una generalización de las capacidades de un grupo etario. El argumento de la simetría sostiene que, como correlato a esa norma de la edad electoral y a la presunción que ella involucra, debe existir otra norma y otra presunción dirigida a excluir a aquellos actuales titulares del derecho a sufragio que han perdido la capacidad necesaria para votar: una edad electoral máxima. Para implementar esta norma, sería necesario un estudio que determine que una mayoría de los sujetos de un grupo etario están seniles o afectos a otras formas de degeneración cognitiva que los hace incapaces electoralmente. Pese a que numerosos miembros de este grupo etario no estarán afectos a la pérdida de su capacidad electoral, su pertenencia a dicho grupo acarreará la pérdida del sufragio de una manera simétrica a la forma en que muchos menores de 18 años son excluidos de las elecciones, pese a que individualmente pasarían cualquier test de capacidad electoral. En este sentido, el argumento de la simetría pretende ser fiel al criterio de la capacidad electoral, siendo insensible a otro tipo de consideraciones (Lau 2012: 864-5).

Hasta aquí, este argumento constituiría una razón para excluir a los ancianos antes que a los menores de edad. El argumento de la simetría, que es una formulación del principio de igualdad de trato, sostiene que los sujetos en semejantes circunstancias deben ser tratados igualmente. Para entender las razones por las cuales los ancianos no son excluidos, uno debe aceptar que, si bien la capacidad electoral es un requisito para otorgar el derecho a sufragio, existen razones adicionales que hacen inconveniente que personas incluidas en 
los registros electorales sean excluidas, pese a haber perdido dicha capacidad. Primero, en el caso de la privación del derecho a voto, el factor simbólico del sufragio tendría preeminencia sobre su dimensión funcional (esto es, su potencial participativo), dado que una posible exclusión acarrearía una degradación de los afectos a ella (Shklar 1991). Segundo, la dimensión funcional también podría pasar a segundo plano, dado que las elecciones admiten la participación individual de numerosos agentes que actúan irracional o desinformadamente. En este caso, las estadísticas arrojarían una previsible insignificancia del número de ancianos cuya capacidad electoral se ha deteriorado en relación con el número de menores de edad políticamente inmaduros (Olsson 2008; Goodin y Lau 2011) ${ }^{1}$. Tercero, en un argumento pragmático de extendido uso, se sostiene que la exclusión de los mayores sería extintiva, esto es 'para siempre', mientras que la exclusión de los menores sería suspensiva. Así, esperar un año o dos no va a trasformar a esos jóvenes en parias, más aún cuando la regla es de carácter universal (Weale 2007: 214).

Más allá del argumento de Lau, la universalización de los estándares de inclusión/exclusión podría servir para sembrar dudas sobre la necesidad de extrapolar el caso de los menores de edad a otros grupos de sujetos que presentan características uniformes que hacen dudar de su capacidad electoral. Entre estos, además de los ancianos, pueden situarse los analfabetos, los sordomudos, los deficientes mentales y, eventualmente, a los ciudadanos adultos no afectos a ninguna de estas cualidades, pero desinformados y/o desinteresados. En contraste con estas potenciales implicancias, la mayor parte de los países que adoptan la fórmula del sufragio universal ofrecen una respuesta clara ante estas dudas. Los miembros de todos estos grupos, y debe recordarse que no siempre ha sido así, son considerados perfectamente capaces para votar. Para ser elector no hace falta saber leer, ni darse a entender por escrito, tampoco es necesario tener interés en política ni haber efectuado estudios básicos, no se requiere ser inteligente, racional o informado. La única regla que cautela la concurrencia de la capacidad electoral es la edad, a lo que debe sumarse —en ciertas jurisdicciones- el hecho de no estar legal o judicialmente incapacitado por razones de salud mental.

Es interesante notar la asimetría que se observa entre esos dos supuestos. Primero, los menores son más radicalmente marginados que las personas con problemas mentales, dado que mientras los segundos normalmente tienen acceso a demostrar su capacidad mediante un procedimiento judicial o administrativo, la presunción que recae sobre los menores no permite prueba en contrario (Munn 2012: 150). Segundo, la incapacidad electoral que es típicamente asociada a las personas con discapacidades mentales severas (por ejemplo, demencia), en términos legales es equivalente a la falta de capacidad 
que afecta a los impúberes (típicamente, menores de 14 años). En contraste, el nivel de desarrollo de esta capacidad — que la regulación exige satisfacer mediante la fijación de la edad electoral a los 18 años- es considerablemente mayor. En este sentido, una persona con una deficiencia mental moderada puede perfectamente votar, mientras que un joven de 17 años con gran interés, racionalidad y conocimiento político resulta ineludiblemente excluido.

Si la idea de universalidad quiere ser tomada en serio y la capacidad electoral ser tenida como un criterio para atribuir el sufragio de forma no-arbitraria, estas asimetrías deben ser corregidas. Las opciones son rebajar el estándar aplicado asimétricamente a los menores de edad, incluyendo cierto grupo de ellos como lo proponen quienes abogan por la reducción de la edad para ejercer el sufragio, o bien mantener dicho estándar pero excluyendo a aquellos mayores de edad que no lo satisfacen. Lo que es claro es que la actual configuración de los criterios de atribución del derecho a voto, entendidos como implementando la noción de capacidad electoral, excluye arbitrariamente a muchos menores de 18 años.

\section{¿Medir la capacidad electoral?}

Los problemas observados hasta ahora no apuntan necesariamente hacia la capacidad electoral como un criterio para extender o restringir el sufragio, sino más significativamente hacia la presunción legal de su ausencia —en el caso de los menores de 18 años- y que no admite prueba en contrario. Si se acepta la hipótesis de que la 'capacidad' es un criterio justificado para excluir a un sujeto de la participación electoral, la presunción de 'incapacidad', basada en un factor no justificado racionalmente (como es el caso del umbral etario), es una exclusión arbitraria. Esta no es una herramienta adecuada para medir dicha capacidad y es, por tanto, no solo una violación del estatus de persona libre e igual de quien se ve afectado (Munn 2012: 141-3), sino una infracción de la regla de argumentación fijada por el principio de sufragio universal en la primera sección de este artículo.

A fin de evitar exclusiones arbitrarias en el uso de la idea de la capacidad electoral, esta podría medirse mediante un test. Cualquiera sea el criterio adoptado (racionalidad, conocimiento teórico o conocimiento pragmático), puede diseñarse una forma de medirla en casos particulares y, de este modo, crear un mecanismo para evitar falsos negativos y falsos positivos. Así, los menores de 18 años podrían acceder al derecho a sufragio cuando puedan demostrar que tienen la capacidad electoral requerida (Olsson 2008: 68). Sin embargo, si lo que se busca es ser fiel a la idea de permitir participar solo a aquellos que demuestren suficiente capacidad electoral, aplicarlo exclusivamente a los menores de 18 años sería también arbitrario, pues implicaría perseverar en la presunción de incapacidad que es justamente lo que se busca evitar con el test. Por otro lado, aplicarlo solo a aquellos que cumplen 18 años sería una dificultad adicional a la presunción de capacidad actualmente vigente. Una vez que se entra en 
el terreno de la evaluación individual de la capacidad electoral, lo más justo sería aplicar el test a todos los potenciales electores y excluir a todos los que no puedan aprobarlo. Además, así como la capacidad electoral se puede adquirir, no resulta descabellado señalar que es algo que también podría perderse. Dicho test de capacidad electoral, siguiendo esta línea de razonamiento, debería ser —además- periódico, para que los actuales y futuros titulares del derecho fueran considerados en iguales términos.

Esta idea no es totalmente diferente a los test que proliferaron para excluir a parte de la población en el pasado y que son defendidos por quienes creen en la epistocracia, o gobierno de los más preparados. Aun cuando puede sonar problemático, si se observa con cuidado, este modelo alternativo no es en realidad demasiado distinto a la actual democracia. Mientras la democracia es epistocráticamente débil, dado que solo excluye a los menores de edad como presuntos incapaces, un régimen fuertemente epistocrático aumentaría el alcance de la exclusión, aunque seguiría basado en el mismo principio: los incompetentes deben ser excluidos. La mayor variación en relación a un régimen democrático consistiría en la alteración de la carga de la prueba. Mientras que el modelo actual de sufragio universal presume la capacidad de los adultos y la incapacidad de los menores, un enfoque epistocrático no presume nada y entrega la habilitación electoral a un test individual y objetivo. De acuerdo con Brennan (2011), este sistema sería mejor que la democracia porque proveería a todos, incapaces incluidos, de un gobierno de mejor calidad (Brennan 2011: 7002; Olsson 2008; Goodin y Lau 2011).

Los problemas que afectan a esta propuesta, sin embargo, son importantes (Munn 2012: 151). Por un lado, el sufragio empoderaría a aquellos que cuentan con adecuados conocimientos y raciocinio. Si se consideran las vinculaciones estadísticas entre éxito académico y nivel de ingresos, tan de moda en estos días, las implicancias prácticas de este modelo, cuando demanda más que un mínimo de racionalidad, apuntarían a una nueva forma de sufragio censitario. Ello implicaría, además, un diametral cambio en la dimensión simbólica del derecho a voto. Este ya no podría servir como criterio de reconocimiento de los miembros libres e iguales que son parte de la comunidad política (Lau 2012: 864), transformándose en un estigma para la vida política y social de las personas excluidas de una práctica de validación social y generando, probablemente, una sociedad de castas. Por otro lado, es claro que no sería fácil, incluso para personas razonables, acordar el contenido del test: algunos abogarían por un test de mínima racionalidad, mientras otros por un test de valores comprometidos con la forma de vida concreta dominante en la sociedad.

Con todo, la literatura ofrece ejemplos más o menos adecuados de test electorales que presentan evidentes ventajas sobre la edad mínima electoral. Por ejemplo, Cook (2013) propone un test mínimo y procedimental, en que los menores puedan acceder al sufragio si se registran por sí mismos. Esto garantizaría, al menos, dos condiciones: que sepan leer y escribir y que puedan actuar con independencia. Por otro lado, Munn (2014) sugiere la implementación de un 
test que sea supletorio a la edad mínima electoral, de modo que sirva únicamente para incluir aquellos menores de 18 años que demuestren capacidad electoral. Estas propuestas implican, sin embargo, que siguen siendo dependientes de la edad mínima electoral a la que transforman en una presunción derrotable. Así, la arbitrariedad se ve limitada pero no eliminada. Por otro lado, al no aplicarse el test universal y periódicamente, sino que solo a los menores de edad, estos siguen siendo discriminados.

\section{Evitar la edad mínima electoral: ¿sufragio para todos?}

Hasta ahora, se ha argumentado que excluir a ciertos sujetos de la participación electoral por razones de capacidad es una práctica odiosa si se implementa mediante la aplicación de un umbral etario. Asimismo, se ha sostenido que existen importantes razones que hacen inconveniente remplazar dicho límite por un test de capacidad y que la arbitrariedad subsistiría aun mediante la aplicación combinada de un test con la subsistencia de la edad mínima electoral. En este escenario, se vuelven atractivas propuestas de carácter más radical que, por contraste, abogan por conceder el sufragio a todos los menores de edad desde el momento de su nacimiento. Olsson (2008), por ejemplo, sostiene que el problema de la capacidad electoral de los menores de edad se termina "si ellos votan ya directamente a través de sus padres o bajo supervisión de sus padres [...]. Con la ayuda de sus padres, los menores de edad tendrán acceso al conocimiento y la experiencia de vida que ellos necesitan para ser un elector tan competente como un adulto" (Olsson 2008: 70). De esta manera, la competencia de los electores siempre estaría garantizada por la presencia orientadora de los padres, al tiempo que todos los menores, en la medida que su desarrollo físico y cognitivo se los permitiera, podrían realizar el acto físico de concurrir a las urnas y depositar el voto.

El principal problema con esta propuesta es que altera el principio democrático de la igualdad política que viene expresado en la idea de una persona un voto, dado que, en la práctica, se estaría entregando más votos a ciertas personas por el solo hecho de tener hijos, como otras propuestas han sostenido más abiertamente (Schrag 2004). Ello, por lo menos, hasta que los menores tengan edad para participar independientemente de sus padres. A esa crítica, Olsson (2008) ha respondido que la relación de representación política entre el padre/madre y el hijo no sería anómala en un sistema basado en la democracia representativa, sustentado a su vez en la representación de los electores por las autoridades. De hecho, los padres representan a sus hijos y toman decisiones tanto o más importantes en casi todas las áreas de la vida. Desde una perspectiva que entiende el sufragio como una herramienta funcional para proteger intereses, esta propuesta resulta razonable, dado que reconoce a los menores como sujetos de intereses y atribuye a su representante legal el poder para concederle autonomía electoral en la medida que vea que el menor desarrolla las aptitudes necesarias para ello (Olsson 2008: 70-3). Sin embargo, como se ha mencionado, 
la noción funcional del voto como expresión de un interés no agota el sentido de nuestras prácticas electorales. Estas reflejan, efectivamente, que el voto es una forma institucionalizada de expresar los intereses individuales en el proceso político, influyendo de manera igualitaria en la formación de las decisiones que nos gobiernan. El voto, no obstante, también representa un reconocimiento del derecho de participar en el autogobierno colectivo como personas libres e iguales. En este último sentido, el voto es un derecho personalísimo de aquellos considerados libres e iguales. Esta dimensión simbólica del sufragio está ligada al reconocimiento, por parte de la sociedad, de cierta capacidad de experimentar la privación de ese derecho como un acto de degradación y exclusión, lo que crucialmente depende de la capacidad de realizar dichos actos de participación de manera autónoma (López-Guerra 2014: 71-7).

Un segundo problema de esta propuesta es que no considera el hecho de que los distintos grupos etarios entre los menores de 18 años son efectivamente muy distintos entre sí (Dahl 1989: 126; Beckman 2009: 114-9). Así, aunque no exista acuerdo respecto a cuál es la edad en que el umbral de capacidad electoral debería ser fijada, existe una importante intuición de que es posible distinguir entre un niño de tres años, el que evidentemente carece de la capacidad necesaria para participar en las elecciones, de un menor de 17 años (Hamilton 2012). Respecto de este, si convenimos que debemos reconocerle capacidad electoral, la representación electoral de sus padres puede convertirse en una limitación de su reconocimiento como un sujeto libre e igual, al menos hasta el momento de la emancipación.

En esta línea, esta propuesta no supone un cambio radical en relación a la arbitraria distinción entre menores y mayores de edad, en la medida que solo el arribo de la mayoría de edad supondría una consecuente prohibición de la intervención de los padres en la decisión electoral de los hijos. La edad mínima electoral, nuevamente, al igual que en el caso de los test electorales, subsiste transformada.

En esta sección del artículo se ha examinado el argumento de la capacidad electoral como justificación de la fijación de la edad electoral. Se han mostrado los problemas que afectan a la fijación de la edad mínima electoral a los 18 años y también que existen buenas razones para rechazar la idea de evaluar la capacidad electoral en términos individuales (dejando fuera a ancianos y otros grupos estimados incapaces). Estos dos supuestos acarrearían, necesariamente, una radical transformación de nuestra comprensión del sufragio como práctica democrática.

Con todo, ello no quiere decir que esas opciones estén perentoriamente descartadas, sino que se encuentran en tensión con los principios democráticos vigentes, especialmente en un régimen de sufragio universal que se construye sobre el principio de igualdad política. 


\section{CAPACIDAD ELECTORAL, CAPACIDAD JURÍDICA Y RECONOCIMIENTO}

En el escenario descrito - y volviendo al problema que se planteó en la introducción-, sería posible sostener que no existen buenas razones para rebajar la edad electoral, dado que, si 18 años es un umbral arbitrario, 16 años no lo sería menos. Al fijar cualquier regla de edad se producirá alguna discordancia entre los sujetos capaces y los habilitados para votar (Hamilton 2012: 1453). Sin embargo, también se ha argumentado que la fijación de una edad mínima electoral, no obstante producir resultados indeseados, es el mecanismo disponible más respetuoso considerando todas las circunstancias. Por ejemplo, afectaría por igual a todas las personas de la misma edad y sería una inhabilidad destinada a terminarse con el tiempo, a diferencia de aquellas que afectan otros grupos excluidos (Cook 2013: 443-4).

Así, existe una razón más poderosa para sostener la rebaja de la edad electoral a, por ejemplo, 16 años. El principio de sufragio universal analizado hablaría a favor de la adopción de la regla más inclusiva, dado que falsos positivos son siempre preferibles a falsos negativos en este rango de edad (López-Guerra 2014: 78-81). Así, la inclusión de todos los menores, incluyendo algunos menores incapaces, estaría justificada si con ello se evita la exclusión de algunos menores capaces.

En esta última sección se argumenta que las razones para conceder el sufragio a ciertos menores de edad son aún más poderosas, dado que existe, al menos, un criterio para justificar la fijación de la edad electoral como expresión de la capacidad electoral.

\section{Capacidad electoral y capacidad jurídica}

La capacidad electoral, se ha argumentado, no es del todo distinta a otro tipo de capacidades que la sociedad reconoce a los sujetos en relación a su desarrollo mental, físico y social. El caso más evidente es el del reconocimiento de la capacidad legal, atendiendo al cual sería perfectamente razonable pensar que una persona que es legalmente responsable debería también serlo política o electoralmente.

El caso de capacidad legal que resulta más obvio para hacer este ejercicio comparativo es el de la capacidad delictual o criminal. En general, los estándares de atribución de responsabilidad criminal son bastante exigentes, por lo que parece ser un ámbito en que tanto los intereses en juego como las consecuencias que se siguen revisten cierto grado de seriedad e importancia (Lau 2012: 866). La edad a la que los sujetos adquieren la capacidad delictual varía considerablemente dependiendo de la jurisdicción. Sin embargo, cuando se examinan las reglas en el Derecho Comparado, puede observarse que esta es consistentemente más baja que la edad electoral. En Chile, por ejemplo, la 
capacidad criminal se adquiere a los 14 años de edad, no obstante aplicársele un estatuto más beneficioso que a los mayores de edad. A los 14 años, por tanto, el sistema jurídico presume que un sujeto tiene el suficiente conocimiento y comprensión del comportamiento que la sociedad espera de él y es consciente de las consecuencias (sanciones) que su conducta podría tener. En contraste, la edad para sufragar se alcanza en Chile recién a los 18 años.

Este argumento comparativo ha sido presentado recientemente por Munn (2012), quien sostiene que la capacidad delictual requiere el mismo tipo de raciocinios y cálculos que la capacidad electoral. Ello evidenciaría que nuestra comunidad tiene parámetros vigentes para indagar sobre este tipo de capacidades. Según este razonamiento, la carga para demostrar que la capacidad electoral es diferente de la capacidad legal - y que estas se adquieren a diferentes edadessería trasferida, recayendo en quienes defienden que la edad electoral debe adquirirse en un momento diferente de la edad legal.

La más obvia crítica a este argumento sostiene que las capacidades electoral y legal atribuyen aptitudes que son diferentes, dado que se aplican en distintas áreas de la vida. La respuesta a este cuestionamiento podría pasar, como lo sostiene Munn (2012: 147-150), por un examen de los test de capacidad electoral que se construyen para evaluar a las personas con deficiencias mentales en ciertas jurisdicciones. Estos test buscan medir similares capacidades de raciocinio que los estándares de capacidad delictual aplicados en las mismas jurisdicciones para medir los niveles de discernimiento de los menores de edad. Así, por ejemplo, ambos requieren (i) conocimiento de la naturaleza de la acción o actividad en cuestión, (ii) comprensión de las consecuencias de participar en tal actividad y (iii) una habilidad para elegir voluntariamente la realización de la acción o actividad. Aquellos menores que han sido considerados criminalmente responsables, han pasado un test de capacidad que sería, al menos, igualmente demandante que uno estándar de capacidad electoral.

No obstante sus fortalezas, este argumento comparativo sufre de los mismos problemas que, como se viera en la sección anterior, afectan al argumento de la capacidad electoral — si es concebido en términos empíricos. En efecto, la misma crítica de arbitrariedad efectuada contra la idea de edad mínima electoral, puede ser hecha en contra de la edad como criterio para fijar la capacidad legal —en general - o responsabilidad criminal —en particular. En estos términos, como se señalara al comienzo del artículo, parece haber un ineludible problema de arbitrariedad asociado a la presunción de capacidad por parte de ciertos grupos etarios, dado que siempre se producirán casos de exclusión e inclusión injustificada.

\section{El aspecto constitutivo de las reglas de capacidad}

El camino que une capacidad electoral y legal ofrece otras posibilidades al argumento comparativo desarrollado por Munn (2012; 2014). Esa vía podría 
encontrarse si, como se sostendrá, existe una conexión interna y no meramente comparativa entre capacidad electoral y legal.

Previo al desarrollo de tal afirmación, sin embargo, debe sostenerse una premisa. Una aproximación entiende que las reglas de capacidad electoral —como es el caso de la fijación de la edad mínima electoral - y toda otra norma que atribuye capacidad para actuar, no solo pretenden regular una realidad existente, sino que también permiten constituir lo que consideramos como capacidad. Con esto no se quiere afirmar que todos los fenómenos de la naturaleza son socialmente construidos, sino solamente que la forma en la que pensamos acerca del mundo está ideológicamente influenciada por medio de la naturalización de ciertas prácticas e instituciones que son socialmente construidas.

En relación a las reglas de capacidad, se pueden distinguir, entonces, dos aspectos concurrentes. Por un lado, la dimensión o aspecto regulativo de los criterios de capacidad dice relación con su posibilidad para intervenir adecuadamente un fenómeno ya existente, al cual ellas pretenden atribuir consecuencias. Así, las reglas de capacidad son susceptibles de ser evaluadas por la conformidad empírica entre la capacidad que se busca demostrar y el mecanismo utilizado para su determinación.

Este aspecto de las reglas de capacidad es el que ha sido depositario de toda la atención de este artículo, siendo el test de capacidad electoral el mecanismo más perfecto para conseguir dicha conformidad y la fijación de la edad electoral uno que, si bien es menos ideal, sería preferible una vez realizado un examen de todas las circunstancias relevantes involucradas. No obstante, una justificación de los requisitos de capacidad que presta atención únicamente a su aspecto regulativo, presenta como problema irremediable, cierto grado de arbitrariedad.

La dimensión o aspecto regulativo de los requisitos de capacidad es una visión parcial de lo que estos son y de cómo son utilizados. Este tipo de reglas también presentan una dimensión o aspecto de reconocimiento, lo que implica que ellas, al mismo tiempo que referirse a la realidad que regulan, contribuyen a configurar dicha realidad. Esta afirmación puede ser objeto de una perspectiva crítica, como la que formula Minow (1990: 9), conforme a la cual dichas reglas de capacidad tienen como efecto distinguir los unos de los otros, el grupo normal del anormal o los individuos autónomos de aquellos que se encuentran en relaciones de dependencia, contribuyendo a asignar categorías que, manifiestamente, se fundan en prejuicios y malentendidos. Así concebidas, las reglas de capacidad serían constitutivas principalmente de relaciones de poder. Sin embargo, no solo la teoría crítica permite entender las reglas de capacidad como reglas constitutivas. Desde otra perspectiva, en abierta tensión con la anterior, los requerimientos de capacidad son formas de establecer expectativas normativas y distribuir responsabilidades mediante el reconocimiento o atribución de ciertos patrones de personalidad a determinados sujetos. Son, en definitiva, expresiones de lo que Klaus Günther (1996) ha llamado, siguiendo a Habermas (1996), una personalidad deliberativa. Esto es, la habilidad general de 
una persona de evaluar acciones de acuerdo con razones y de actuar en relación con aquellas razones que acepta. Esta es la idea de capacidad presupuesta en la noción de sujeto de derecho.

La ventaja de reconocer que las reglas de capacidad no solo regulan, sino también reconocen a los sujetos como capaces e incapaces, está en entender que tales habilidades son atribuidas, adscriptas o reconocidas a los sujetos, y no son, por el contrario, solo objeto de juicios de verificación o comprobación. Si asumimos que los requerimientos de capacidad no son solo regulativos, sino también implican el reconocimiento de ciertas circunstancias que son adscritas a ciertos individuos, hemos de admitir que aquellos que son excluidos no lo son necesariamente porque carecen de ciertas características, sino que su exclusión, de cierta manera, articula una diferencia respecto de quienes son considerados capaces. La selección de las características requeridas implica ciertos juicios y decisiones. Esto tiene, como consecuencia, que las incapacidades pueden ser vistas como categorías políticas que, si bien manifiestan una faz de exclusión y dominación (Minow 1990: 124), al mismo tiempo están abiertas a ser utilizadas en un sentido emancipatorio para criticar la exclusión de ciertos sujetos o reivindicar su inclusión (Mouffe 1999: 43-4; Balibar 2004: 59-60).

Esa dimensión de reconocimiento se ve refrendada cuando observamos cómo estos requisitos operan en el nivel del sistema jurídico, por ejemplo, cuando se advierte cómo los adultos son considerados legalmente capaces y, por tanto, legalmente responsables - aun cuando, en un caso concreto, un adulto pueda mostrar comportamientos y actitudes solo compatibles con una persona que carece las habilidades cognitivas o los conocimientos considerados como necesarios para desempeñar una actividad para la cual, en razón de su adultez, se reputa capaz. En estos casos, si bien las expectativas cognitivas establecidas por las normas de capacidad pueden verse defraudadas, las expectativas normativas, por regla general, perseveran en comprender a dichos sujetos como capaces y, por tanto, responsables. Otro tanto pasa en el caso de los menores de edad.

\section{La capacidad electoral como expresión de reconocimiento democrático}

Una vez establecido el carácter de reconocimiento de las reglas de capacidad, un segundo paso en el argumento que quiere presentarse dirige su atención al rol social de las normas de capacidad. Estas, debe observarse, son presupuestos de una sociedad racionalmente organizada. El tipo de capacidades asociadas a lo que se ha llamado personalidad deliberativa, es reconocido a sus miembros de manera general y no específicamente. Ello viene dado por el hecho que, de esa generalidad, depende la posibilidad de atribución de responsabilidad a los agentes y la coordinación de intereses y esferas de autonomía sobre las que la organización social moderna se construye. 
Mientras este reconocimiento general obra 'por defecto', la personalidad deliberativa es solo excluida, y la falla de las expectativas cognitivas es seguida de un ajuste en las consecuencias normativas de manera excepcional. Por ejemplo, cuando una persona daña a otra en su propiedad, ella es — por defectoconsiderada responsable y solo queda excluida de dicha responsabilidad si puede demostrarse que ella no actuaba conforme a razones autónomamente determinadas, esto es, por ejemplo, bajo amenaza de muerte o afectada por una deficiencia mental severa.

A este respecto, la constitución de los menores de edad como sujetos legalmente incapaces puede explicarse por el hecho de que la comunidad les niega personalidad deliberativa en cuantos sujetos de derecho. Por otro lado, si la falta de reconocimiento de capacidad electoral tiene un correlato a una falta de reconocimiento en otros aspectos de su personalidad deliberativa, la exclusión de los menores de edad de los procedimientos electorales puede ser considerada como coherente con su posición dentro de la sociedad. Así, la constitución de los menores de edad como sujetos electoralmente incapaces permite explicar que la comunidad les niega la personalidad deliberativa en cuanto ciudadanos, mientras también les niega, al menos parcialmente, en cuanto sujetos de derecho.

Lo anterior tiene relevancia para una crítica a la fijación de la edad electoral en la siguiente medida. La expectativa de comportamiento moral autónomo asociado a la personalidad deliberativa no puede dirigirse solamente a la constitución de los menores de edad como sujetos de derecho, sino que debe, a su vez, estar relacionada también con su constitución como ciudadanos. La configuración de las esferas de autonomía privada y pública están basada, en último término, en el mismo estatus moral, y no pueden, por tanto, ser radicalmente disociadas (Mañalich 2011: 124-5). Ello se muestra especialmente relevante en el contexto de un régimen democrático donde, por un lado, la atribución de derechos de participación viene a justificar la expectativa de adaptación del comportamiento a las normas de conducta determinadas autoritativamente por la comunidad, a través de procedimientos democráticos abiertos a los mismos sujetos en cuanto ciudadanos (Günther 1996: 1057).

Por otro lado, si ciertas personas son consideradas con la capacidad de adecuar su comportamiento a las expectativas normativas como sujetos de derecho, esos mismos sujetos deberían ser reputados como capaces para participar en el proceso conducente al establecimiento de las normas que fijan dichas expectativas. De otra manera, la justificación de la participación democrática perdería su relación, a su vez, con la justificación de la legitimidad del derecho al cual las personas están vinculados incondicionalmente en cuanto sujetos de derecho. Esta relación interna entre los conceptos de ciudadano y sujeto de derecho, que en una democracia representativa se articula principalmente mediante la atribución del derecho a voto a aquellos cuya conducta se pude esperar se adapte al derecho - que podría denominarse una relación de reconocimiento democrático-, tiene su expresión en cierta coherencia entre 
las reglas de capacidad que administran, y de cierta manera atan, las esferas jurídica y política.

Así, cuando se atiende a esta concepción normativa de la persona, surge una nueva perspectiva desdela cual puedenjuzgarse los requerimientos de capacidad electoral. Lo que se busca no es la coincidencia entre las capacidades empíricas y el estatus normativo de la persona, sino el tratamiento normativo coherente de dichas personas en todos los aspectos relevantes de su personalidad. No puede tratarse a una persona como capaz en ciertas esferas y como incapaz en otras que envuelven el ejercicio de una capacidad crítica similar sin una justificación. Por ejemplo, parece completamente anómalo que sujetos que son considerados plenamente responsables para cometer delitos no posean la capacidad legal para conducir sus negocios civiles de manera independiente.

Por tanto, un importante elemento para criticar una determinada fijación de la edad electoral es la comparación con la edad en que se realiza la atribución de derechos y responsabilidades en la esfera legal, una realidad que puede observarse en la esfera de los negocios y del trabajo, pero especialmente, de la justicia criminal. Considerar este elemento reivindica una presunción en favor de la fijación de una edad electoral no mayor a la edad en que se adquiere responsabilidad legal.

Considerando que los menores de 18 años y mayores de 16 años son considerados como capaces en importantes y numerosas esferas de la vida social - en particular si se considera que, generalmente, dichos sujetos son tenidos como criminalmente responsables y tienen capacidad civil de administrar ciertos bienes, de testar, de reconocer a un hijo y de hacerse responsables extracontractualmente por daños-, al no ser autorizados para ejercer derechos políticos se produce una violación de la expectativa de ser reconocidos como sujetos capaces formar juicios morales propios (Honnett 1995:134).

Antes de concluir, debe adelantarse una respuesta a algunas objeciones que, además, pueden contribuir a explicar mejor el argumento aquí sostenido. Se podría afirmar que, dado que los menores a los que se estaría concediendo el sufragio se encuentran bajo un estado de incapacidad civil, típicamente bajo la patria potestad de sus padres, resulta paradójico concederles plena capacidad electoral si el sistema jurídico no les reconoce plena capacidad legal y los somete a la voluntad de un adulto que supervisa su actuar y toma decisiones soberanas sobre su vida.

La misma idea de que autonomía privada y pública son co-originales y están mutuamente implicadas, serviría de soporte a esta crítica. Frente a esto, puede responderse que el argumento para la rebaja de la edad electoral puede tomar la forma, con las necesarias adaptaciones y especificaciones, de un argumento para la disminución de la edad de emancipación. Sin embargo, bajo las actuales circunstancias, en que los menores de 18 son tenidos como incapaces relativos, 
una respuesta satisfactoria debe dirigirse a explicar y justificar la asimetría entre dichas capacidades.

Al sostener que la capacidad política debería entenderse adquirida por aquellos a los que el sistema jurídico reconoce capacidades similares, se asume que debe haber cierta coherencia entre los regímenes de capacidad. No se sostiene, sin embargo, una identidad. Una respuesta adecuada debe pasar por reconocer los rasgos institucionales y las consecuencias individuales de los derechos que son objeto de cada uno de estos dominios. Mientras que para justificar la autonomía relativa de los menores en el ámbito civil siempre se ha ofrecido el argumento de su propia protección, su exclusión de las elecciones no puede entenderse de la misma manera. En general, la incapacidad civil es parcial y tiene como fundamento evitar el daño tanto para terceros como para los propios menores de edad. La incapacidad electoral es total, no admitiendo graduaciones ni excepciones, y la participación de los menores no produciría un impacto negativo en la calidad de los resultados electorales ni perjudicaría a los propios menores (Beckman 2009: 114-9).

Sobre esta base, también podría responderse a quienes sostienen que afirmar esta coherencia involucraría la legitimación de la responsabilidad penal juvenil mediante la incorporación de ciertos menores de edad en el padrón electoral. La responsabilidad penal, en estos términos, no podría ser menor a la edad electoral, pero sí podría ser mayor, atendiendo a consideraciones de vulnerabilidad y a las necesidades especiales de cuidado de estos sujetos.

Una segunda objeción sugiere que este argumento, que encuentra su foco en la coherencia de la regulación de las capacidades reconocidas a los sujetos en un contexto social, político y jurídico determinado, privaría de la posibilidad de criticar la fijación de un umbral de capacidad que muestre coherencia en las esferas política y jurídica. Esto sucedería, por ejemplo, si como sociedad convenimos un umbral de capacidad general a los 18 años, que rija tanto para la esfera legal como para la electoral. El argumento aquí defendido tendría, como consecuencia, silenciar las críticas de aquellos menores de 18 años que demandan inclusión electoral desde una perspectiva regulativa, por ejemplo, por considerar que tienen las mismas cualidades cognitivas y cuentan con la misma información relevante para una elección informada que los mayores de 18 años.

Esta objeción es importante, pero solo parece ser dirigida a una concepción acerca de las reglas de capacidad que no admite un aspecto regulativo y que las comprende solamente en su dimensión de reconocimiento. Llamar la atención sobre el aspecto de reconocimiento de estas reglas no quiere decir que esta dimensión no tenga relación alguna con la dimensión regulativa y, por tanto, con cuestiones empíricas. Estos aspectos están en una relación de mutua dependencia, debido a que cuestiones empíricas son siempre socialmente aprendidas y construcciones sociales son siempre empíricamente influenciadas. Bajo esa premisa, la aproximación aquí defendida no busca abrir un nuevo 
frente (coherencia normativa/reconocimiento democrático) desde el cual criticar a las actuales reglas de edad mínima electoral a costa de cerrar otro frente (arbitrariedad empírica) desde el cual puedan criticarse.

Por un lado, no busca necesariamente negar la arbitrariedad de la fijación de la edad electoral conforme a criterios empíricos. Sin embargo, la eliminación de la arbitrariedad empírica (se señaló en la sección anterior) no puede lograrse a costa de la masiva afectación de la integridad de la elección o la igualdad política. Por otro lado, la referencia al aspecto de reconocimiento de las reglas de capacidad implica la admisión de que estas normas no son naturales, sino que son socialmente construidas y, por tanto, pueden ser objeto de crítica. Son - como toda otra forma de exclusión social, legal y política- de ciertos sujetos, un espacio central de la política democrática. El argumento desarrollado en esta sección es un ejemplo, asociado a una determinada perspectiva, de las posibles críticas a las actuales reglas de capacidad electoral, pero ciertamente no la única disponible.

\section{CONCLUSIÓN}

La edad mínima electoral es una cuestión crecientemente discutida, por lo que es relevante explorar sus fundamentos. Uno de los problemas principales relacionados con ella es que la inclusión de los menores de edad debe ser coherente con el resto de la práctica de inclusión electoral. En este sentido, el análisis debe partir del supuesto de que el sufragio universal exige incluir a todos aquellos sujetos cuya exclusión no esté debidamente justificada. En este contexto, una de las principales razones que se han ofrecido para justificar la exclusión de los menores apunta a que estos no son competentes para participar en las elecciones.

Si bien la mayor parte de la literatura se concentra en demostrar que los menores poseen o carecen de ciertos rasgos racionales o ciertos conocimientos considerados como relevantes para participar correctamente en las elecciones, las formas de asegurar que los votantes participen en la elección correctamente se limitan, en la práctica, a excluir a menores de edad y a personas con serias discapacidades mentales. Al someter esta práctica al estándar de justificación que exige el principio de sufragio universal, arroja la conclusión que, al menos algunos menores de edad, estarían excluidos arbitrariamente.

El criterio según el cual hemos de definir el umbral de inclusión debería recurrir a dos ideas que han sido desarrolladas en este artículo. En primer lugar, entender que la regulación electoral no adopta una pauta coherente respecto al concepto de capacidad electoral, lo que ha llevado a la conclusión que dicha exclusión es arbitraria. Ello no implica, necesariamente, afirmar que ciertos menores no deban ser excluidos o que la noción misma de capacidad electoral debe ser evitada. 
En segundo lugar, si los menores son considerados como responsables para cometer delitos y celebrar ciertos actos civiles, resulta extraño que un estándar de capacidad más demandante sea la excusa para marginarlos de las elecciones. En ese sentido, el umbral de atribución de la capacidad legal puede ser una herramienta importante en la búsqueda de un umbral coherente de atribución de capacidad electoral. En este ejercicio comparativo, sin embargo, no debe pensarse en la capacidad legal como prueba de la existencia de ciertas facultades, sino como la expresión de que una comunidad democrática presupone dichas facultades por defecto y reconoce a determinados sujetos como titulares de ciertos derechos y responsabilidades, tanto en la esfera legal como en la política.

Se puede concluir, por tanto, que, bajo ciertas circunstancias, la inclusión de algunos menores de edad es requerida. Aunque este argumento no permite concluir exactamente cuál es la edad más adecuada para fijar una regla de edad mínima electoral, la mantención de la exclusión de los mayores de 16 años resultaría particularmente injustificada si esta se encuentra disociada de la edad en que cierta capacidad legal es reconocida a los menores de edad.

Teniendo estas conclusiones teóricas a la vista, podría sostenerse que las propuestas de modificación de la regulación electoral que pretenden rebajar la edad electoral a 16 años, no resultarían problemáticas.

\section{REFERENCIAS}

Balibar, Etiene. 2004. We, the People of Europe? Reflexions on Transnational Citizenship. Princeton: Princeton University Press.

Beckman, Ludwig. 2007. "Who Should Vote? Conceptualizing Universal Suffrage in Studies of Democracy". Democratization 15(1): 29-48.

Beckman, Ludwig. 2009. The Frontiers of Democracy. The Right to Vote and its Limits. London: Palgrave Macmillan.

Beckman, Ludwig. 2014. "The Accuracy of Electoral Regulations: The Case of the Right to Vote by People with Cognitive Impairments". Social Policy and Society 13(2): 221-233.

Blais, André; Louis Massicotte y Antoine Yoshinaka. 2001. "Deciding Who has the Right to Vote: A Comparative Analysis of Election Laws". Electoral Studies 20: 41-62.

Brennan, Jason. 2011. "The Right to a Competent Electorate". The Philosophical Quarterly 61: 700-724.

Caciagli,Mario. 2009. "El Debate del Voto a los 16 años en Italia". Revista de Estudios de la Juventud 85: 1-8.

Cook, Philip. 2013. "Against a Minimum Voting Age". Critical Review of International Social and Political Philosophy 16(3): 439-458.

Cowden, Mhairi (2012): "Capacity, Claims and Children's Rights". Contemporary Political Theory 11: 362-380.

Dahl, Robert. 1989. Democracy and its Critics. New Haven: Yale University Press.

Dworkin, Ronald. 1978. Taking Rights Seriously. Cambridge: Harvard University Press.

Ewald, Alex. 2004. "An 'Agenda for Demolition': The Fallacy and the Danger of the 'Subversive Voting' Argument for Felony Disenfranchisement". Columbia Human Rights Law Review 36: 109-143.

Goodin, Robert y Jane Lau. 2011. “Enfranchising Incompetents: Suretyship and the Joint Authorship of Law". Ratio 24(2): 154-166. 
Günther, Klaus. 1996. "Communicative Freedom, Communicative Power, and Jurisgenesis". Cardozo Law Review 17(4-5): 1035-1058.

Habermas, Jurgen. 1996. Between Facts and Norms. London: Polity Press.

Hamilton, Vivian. 2012. "Democratic Inclusion, Cognitive Development, and the Age of Electoral Majority". Brooklyn Law Review 77(4): 1449-1516.

Hart, Daniel y Robert Atkins. 2011. "American Sixteen- and Seventeen-Years-Olds are Ready to Vote". The Annals of the American Academy of Political and Social Science 633(1): 201222.

Honneth, Axel. 1995. The Struggle for Recognition: The Moral Grammar of Social Conflicts. Cambridge: The MIT Press.

Lau, Jane. 2012. "Two Arguments for Child Enfranchisement". Political Studies 60(4): 860-876.

López-Guerra, Claudio. 2010. "Enfranchising Minors and the Mentally Impaired". Social Theory and Practice 38(1): 115-138.

López-Guerra, Claudio. 2014. Democracy and Disenfranchisement: The Morality of Electoral Exclusions. Oxford: Oxford University Press.

Mañalich, Juan Pablo. 2011. "Pena y ciudadanía”. En Pena y culpabilidad en el Estado democrático de derecho, editado por Urs Kindhäuser y Juan Pablo Mañalich. Buenos Aires: Editorial BdF.

Minow, Martha. 1990. Making All the Difference: Inclusion, Exclusion and American Law. Ithaca: Cornell University Press.

Mouffe, Chantal. 1999. "Carl Schmitt and the Paradox of Liberal Democracy". En The Challenge of Carl Schmitt, editado por Chantall Mouffe. London: Verso.

Munn, Nicholas. 2012. "Reconciling the Criminal and Participatory Responsibilities of the Youth". Social Theory and Practice 38(1): 139-159.

Munn, Nicholas. 2014. "Capacity Testing as a Means of Increasing Political Inclusion". Democratization 21(6): 1134-1152.

"The Disenfranchisement of Ex-Felons: Citizenship, Criminality, and 'The Purity of the Ballot Box"'. 1989. Harvard Law Review 102(6): 1300-1317.

Olsson, Stefan. 2008. “Children's Suffrage: A Critique of the Importance of Voters' Knowledge for the Well-Being of Democracy". The International Journal of Children's Rights 16(1): 55-76.

Schrag, Francis. 2004. "Children and Democracy: Theory and Policy". Politics, Philosophy \& Economics 3(3): 365-379.

Shklar, Judith. 1991. American Citizenship: The Quest for Inclusion. Cambridge: Harvard University Press.

Wagner, Markus, David Johann y Sylvia Kritzinger. 2012. “Voting at 16: Turnout and the Quality of Vote Choice". Electoral Studies 31(2): 372-383.

Weale, Albert. 2007. Democracy. London: Palgrave Macmillan.

Wintersberger, Helmut. 2007. "Edad Electoral, dieciséis años. La Reforma Electoral Austriaca de 2007". Revista de Estudios de la Juventud 85.

Winkler, Adam. 1993. “Expressive Voting”. New York University Law Review 68: 330-388.

Pablo Marshall es profesor de Derecho de la Universidad Austral de Chile. Es doctor en Derecho por la Universidad de Glasgow y licenciado en Derecho por la Universidad de Chile. Es además editor de la revista académica Derecho y Crítica Social.Correo electrónico pmarshall@uach.cl 